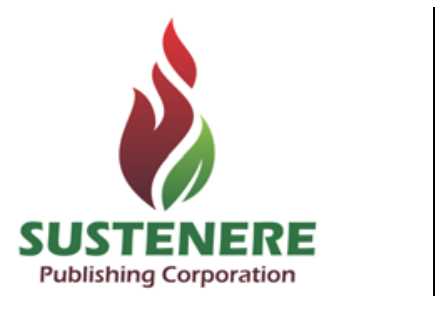

\section{ANÁLISE DE REDES SOCIAIS PODE APROXIMAR GOVERNO E CIDADÃO?}

\section{RESUMO}

O compartilhamento de ideias, opiniões e experiências dos usuários é cada vez mais frequente em redes sociais on-line. Esse comportamento pode transformar tais ambientes em fontes de informações valiosas, pois neles a produção de conteúdo é descentralizada e, via de regra, não há censura. $A$ análise de redes sociais refere-se ao processo de coletar, medir, analisar e interpretar resultados de associações e interações entre usuários e tópicos dentro de redes sociais, de forma a identificar opiniões, avaliar o que está sendo discutido, descobrir o sentimento de um usuário sobre um tema, apontar tendências e, inclusive, prever situações que poderão ocorrer. Cada vez mais as empresas privadas utilizam técnicas e ferramentas de análise de redes sociais para entender e acompanhar o comportamento de seus clientes. Em paralelo, exemplos de análises de redes sociais on-line aplicadas ao tema governo começam a surgir. Este artigo exemplifica como a análise de redes sociais pode ser um instrumento importante para aproximar governo e cidadãos, permitindo identificar opiniões de grupos de cidadãos sobre assuntos governamentais ou organização de eventos de interesse da sociedade, por exemplo. Para isto, e apresentado um estudo de caso em que foram geradas visualizações a partir de dados extraídos das redes sociais Twitter e Facebook durante a Copa do Mundo da Fifa Brasil 2014. O artigo expõe os resultados e insights obtidos do estudo de caso e o aprendizado alcançado com esta experimentação, incluindo os passos realizados e as dificuldades encontradas. O objetivo principal do trabalho foi demonstrar o potencial de análise de redes sociais na compreensão de opinião pública sobre um assunto de interesse do Estado, contribuindo assim para a aproximação de governo e cidadão.

PALAVRAS-CHAVES: Análise de Redes Sociais; Governo; Visualização de Informação.

\section{CAN SOCIAL NETWORK ANALYSIS BRING GOVERNMENT AND CITIZENS CLOSER TOGETHER?}

\section{ABSTRACT}

Sharing ideas, opinions and experiences of users is increasingly frequent in on-line social networks. This behavior can turn such environments in valuable sources of information, especially because the content production is decentralized and, as a rule, there is no censorship. Social network analysis refers to the process of collecting, measuring, analyzing and interpreting results of interactions and associations among users and topics within social networks in order to identify opinions, evaluate what are the subjects being discussed, find users' feeling about a topic, identify trends and even anticipate situations that may occur. Increasingly, private companies use social network analysis to understand and monitor their customers' behavior. At the same time, examples of on-line social networking analysis regarding government themes are emerging. This article exemplifies how social network analysis can be an important tool to bring government and citizens closer together, allowing to identify opinions about government affairs or to organizing events of interest to society, for instance. For this, we present a case study that analyses data extracted from Twitter and Facebook social networks during the FIFA World Cup Brazil 2014. This article reports results and insights from the case study and brings lessons learned during the experiment, including steps performed and difficulties found. This work's main goal was to demonstrate the potential of social network analysis in understanding public opinion on a matter of national interest, thus contributing to approach of government and citizen.

KEYWORDS: Social Network Analysis; Government; Information Visualization.

\section{Revista Brasileira de}

Administração Científica,

Aquidabã, v.5, n.2, Out 2014.

\section{ISSN 2179-684X}

\section{SECTION: Articles}

TOPIC: Sistemas e Tecnologia da

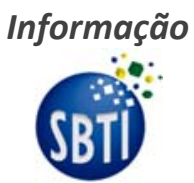

Anais do Simpósio Brasileiro de Tecnologia da Informação (SBTI 2014)

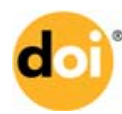

DOI: 10.6008/SPC2179-684X.2014.002.0013

José Ronaldo Agra de Souza Filho Universidade Federal de Campina Grande, Brasil http://lattes.cnpq.br/1699088468619335 ronaldoagra@gmail.com

Fernanda Santos Fonseca

Universidade Salvador, Brasil http://lattes.cnpq.br/4988172237909504 nandasf88@gmail.com

\section{Rodrigo Hjort}

Serviço Federal de Processamento de Dados, Brasil http://lattes.cnpq.br/8248807637318409 rodrigo.hjort@gmail.com

Viviane Dias Malheiros de Pinho Serviço Federal de Processamento de Dados, Brasil http://lattes.cnpq.br/7939197170515112 vivianedmp@yahoo.com

Received: 31/08/2014

Approved: $15 / 10 / 2014$

Reviewed anonymously in the process of blind peer

\section{Referencing this:}

SOUZA FILHO, J. R. A.; FONSECA, F. S.; HJORT, R. PINHO, V. D. M.. Análise de redes sociais pode aproximar governo e cidadão?. Revista Brasileira de Administração Científica, Aquidabã, v.5, n.2, p.172185, 2014. DOI: $h$ ttp://dx.doi.org/10.6008/SPC2179$684 X .2014 .002 .0013$ 


\section{INTRODUÇÃO}

A troca de informações e opiniões entre pessoas, sobre temas variados, é cada mais intensa em redes sociais on-line. Cerca de $77 \%$ dos usuários da Internet usam redes sociais durante sua navegação na Web (CETIC, 2013). Redes sociais on-line permitem ao usuário conectar-se com pessoas distantes; conhecer usuários com interesses em comum; e compartilhar sentimentos e pensamentos; conferindo voz a indivíduos que se tornam, também, produtores de informação.

Em uma rede social on-line, a produção de conteúdo é descentralizada e, via de regra, não há censura. Tais redes facilitam a comunicação e mobilização de pessoas, permitindo que grupos se organizem e estruturem de forma fácil e rápida. Exemplo disto, foram as manifestações durante a Copa das Confederações no Brasil em 2013 (FRANÇA \& OLIVEIRA, 2014), muitas vezes organizadas a partir de redes sociais. Dessa forma, as redes sociais on-line têm se tornado fontes importantes para que empresas e governos alinhem suas decisões com as expectativas de seu público-alvo.

A análise social é o processo de coletar, medir, analisar e interpretar resultados de associações e interações entre pessoas e ideias dentro de redes sociais. Para apoiá-la, são empregadas técnicas especializadas, tais como: análise de sentimentos, processamento de linguagem natural, análise de texto, modelagem preditiva e recomendações, além de identificação automatizada e classificação de assunto/tema, pessoas ou conteúdo.

Este artigo apresenta como a análise de redes sociais pode ser um instrumento facilitador para aproximar governo e cidadãos, ajudando a identificar e analisar a opinião pública - sobre programas de governo e o seu trabalho - e possibilitando, aos gestores públicos, atender melhor às necessidades da sociedade. Para demonstrar o potencial de análise de redes sociais na compreensão de comentários e mensagens de cidadãos sobre um assunto, este artigo apresenta um estudo de caso realizado durante o período da Copa do Mundo da Fifa Brasil 2014. Neste estudo, publicações das redes sociais Twitter e Facebook sobre os temas Copa do Mundo e Governo foram coletadas, e diversas análises sobre elas foram realizadas - incluindo o seu conteúdo, os usuários que as efetuaram, as hashtags empregadas e os usuários mencionados.

Este trabalho está organizado da seguinte forma, após esta introdução: a segunda seção "Análise de Redes Sociais" apresenta conceitos básicos sobre análise de redes sociais e as atividades típicas para sua realização; a terceira seção expõe trabalhos relacionados ao uso de análise de redes sociais em temas de interesse de governos; a quarta seção discute o estudo de caso realizado; e a quinta seção "Resultados do estudo de caso" exemplifica insights a partir da aplicação de técnicas de análise aos dados coletados. Por fim, a última seção "Considerações Finais" contém considerações sobre o estudo e trabalhos futuros. 


\section{REVISÃO TEÓRICA}

\section{Análise de Redes Sociais}

Diversas redes sociais on-line foram criadas nos últimos anos, com uma grande quantidade de informações em tempo real. Fóruns, blogs, redes sociais de relacionamento e sites de exibição de imagens e vídeos são exemplos de redes sociais que servem como fontes de informações para análise. Algumas vezes, notícias e acontecimentos são apresentados em redes sociais pelos seus usuários antecipando-se à imprensa convencional. O fluxo de informações nas redes sociais é atualizado pelo próprio usuário, trazendo informações pessoais dos indivíduos e opiniões, via de regra, sem censura, sobre uma variedade de temas.

A análise de redes sociais envolve os seguintes processos técnicos: (i) coleta de dados das redes; (ii) tratamento dos dados; (iii) análise, incluindo a descoberta de informação; e (iv) apresentação dos resultados.

A coleta consiste em extrair dados brutos relevantes das redes sociais. Nesta etapa, são obtidos dados sobre pessoas e organizações (entidades), incluindo: conversas, conexões, interações e informações compartilhadas por uma entidade em uma rede social. O volume de dados de uma rede social é relativamente grande, e por isso é comum, durante a coleta, aplicar filtros por assunto ou característica dos publicadores ("postadores") e publicações ("postagens") para obter dados contextualizados - e, consequentemente, em menor quantidade.

O tratamento dos dados brutos consiste em organizá-los, limpá-los e enriquecê-los. Técnicas para tratar o dado podem ser: processamento de linguagem natural (identificação de entidades, temas e tópicos, classificação de texto); serviços de geolocalização; identificação de idiomas; entre outras. Ao final do tratamento, tem-se uma base limpa e enriquecida com metadados e dados agregados que complementam os dados brutos coletados.

A próxima etapa é a análise propriamente dita, quando os dados são analisados de forma agregada e as relações entre os dados são exploradas. Várias técnicas de análise podem ser utilizadas para entender o que está sendo comentado nas redes sociais, como: análise de tendências; análise de vínculos (quem se relaciona com quem e porquê); análise geoespacial; e análise de sentimentos (avaliação positiva x negativa). Assim, é possível responder a questões como: ‘Qual tema é tendência nas redes sociais?', 'Quem são os influenciadores neste tema?”, “O sentimento em relação ao tema é positivo ou negativo?'.

Análise de redes sociais (WASSERMAN \& FAUST, 1994) refere-se a um conjunto de técnicas especialmente interessante para a análise social, pois consiste no estudo das interações entre os atores de uma rede. Uma rede social pode ser representada na forma de um grafo, onde os nós da rede são os atores que representam pessoas. As ligações entre as pessoas são as relações ou fluxos de informações entre os nós. Além da utilização dos conceitos relacionais, alguns princípios centrais subjacentes à perspectiva de rede são: atores e suas ações são vistos como 
interdependentes; as ligações entre atores são canais para transferência de recursos; os modelos de rede focados nos indivíduos veem a estrutura da rede como meio de oportunidades ou restrições para as ações dos indivíduos; modelos de redes podem conceituar estruturas sociais, econômica, política, dentre outras entre os indivíduos (WASSERMAN \& FAUST, 1994). As análises podem ser feitas em relação à posição de um indivíduo na rede e suas relações - identificar se ele é um líder ou um ator isolado - ou à visão da rede ou sub-redes com um todo.

A etapa de apresentação oferece a visualização das informações tratadas e analisadas (dashboards e relatórios), de forma a facilitar a identificação de padrões, tendências e informações relevantes. Várias técnicas de visualização - dentre as quais podemos citar treemapping (VLIEGEN, 2006) e mapas temáticos (SLOCUM, 2008) - podem ser utilizadas para complementar a análise e subsidiar novas descobertas, em um processo conhecido como mineração visual (INSELBERG, 1998).

Várias soluções de mercado implementam uma ou mais destas etapas (coleta, tratamento, análise e visualização). Para a coleta, é possível utilizar APIs para acesso direto às redes sociais; ou serviços agregadores, que entregam grandes volumes de dados quase "em tempo real", além de dados históricos e informações extras, como geolocalização. Assim, tais serviços fazem também parte do tratamento. As APIs específicas têm a vantagem de serem gratuitas e a desvantagem de depender da construção de extratores específicos para cada rede social. As etapas de análise e visualização são, muitas vezes, fornecidas pela mesma solução. As opções disponíveis no mercado diferenciam-se em abrangência e especialização. Existem soluções especializadas em domínios como marketing ou fraudes - e soluções generalistas, flexíveis para diferentes domínios. Rozwell (2014) consolidou uma visão inicial de opções do mercado.

Não só a iniciativa privada pode lograr vantagens com a análise de redes sociais, mas também o serviço público. Como as redes sociais se tornaram um local onde os usuários expressam e compartilham suas opiniões livremente, o governo pode, a partir delas, obter opiniões honestas sobre os mais diversos assuntos. É mais uma forma de aproximar o governo e a sociedade. Alguns governos já usam ferramentas de análise social, como ilustrado nesta seção.

Segundo ONG (2014), no governo de Cingapura, ferramentas são utilizadas para: (i) analisar o sentimento das pessoas para formular políticas públicas novas; (ii) proteger a reputação do país, monitorando fóruns e blogs de investidores e turistas para conhecer o ambiente necessário para negócios e turismo; (iii) melhorar a resposta a desastres - buscando por sintomas de doenças para detectar possíveis epidemias, ou analisando a situação após um desastre para priorizar a alocação de recursos de emergência; e (iv) avaliar e mitigar crises potenciais, por meio de análise de agitação social, identificação de informações falsas, e medidas para dissipar rumores.

$\mathrm{Na}$ cidade de Toulouse - França, um experimento realizado pela prefeitura analisou redes sociais e publicações relacionadas a necessidades públicas dos cidadãos (IBM, 2014). A análise incluiu fatores como contexto, conteúdo e sentimento das publicações. Os resultados da análise serviram para priorizar e entender os anseios da população e, com isso, melhorar o planejamento 
e gerenciamento dos problemas da cidade e melhorar a imagem do governo para os cidadãos (IBM, 2014).

$\mathrm{Na}$ Irlanda, um estudo sobre a análise de redes sociais foi feito para apoiar a compreensão de mudanças nas condições de trabalho (UNITED NATIONS, 2014). Foram analisados humores e temas presentes nas conversas relacionadas com o desemprego.

No Brasil, pesquisadores da Universidade Federal de Minas Gerais (UFMG) criaram observatórios para monitorar e visualizar como um tema está sendo veiculado em mídias sociais variadas. Dessa forma, foi possível avaliar o impacto de campanhas na Internet e verificar como os usuários interagem e reagem às notícias e discussões. Outros exemplos de aplicação da UFMG são: o Observatório da Copa do Mundo e o Observatório da Dengue. Pesquisadores da Universidade Federal do Rio de Janeiro (UFRJ) publicaram trabalhos relacionados à análise de sentimentos das pessoas (apoio ou repúdio) (FRANÇA e OLIVEIRA, 2014) e à identificação de influenciadores (THEODORO et al., 2014) durante os protestos e manifestações que ocorreram no Brasil em 2013. Os dados do trabalho foram coletados do Twitter. Por sua vez, pesquisadores da Universidade Federal do Rio Grande do Sul (UFRGS) identificaram "aspectos"1 de candidatos eleitorais em comentários de notícias (SAPIRAS \& BECKER, 2014). Acredita-se que a mesma técnica para identificar o sentimento em relação a aspectos de governo, que foi aplicada para comentários de notícias, poderá ser aplicada a publicações de redes sociais on-line. Para ter-se uma ideia de como estão crescendo as discussões em torno do uso de análise de redes sociais para compreender temas de interesse do governo; "Sentimentos, Protestos e Eleições" foi o tema principal de uma seção técnica no III Brazilian Workshop on Social Network Analysis and Mining, em 2014.

\section{METODOLOGIA}

O estudo de caso realizado consistiu em coletar, tratar e analisar dados provenientes das redes sociais Twitter e Facebook, que correlacionassem os temas 'Copa do Mundo' e 'Governo'. O objetivo principal do estudo foi identificar o potencial de analisar a opinião pública e conhecer o comportamento de usuários em redes sociais on-line para um assunto relacionado a governo. Objetivos secundários foram observar as dificuldades relacionadas a cada fase do processo de análise e experimentar técnicas e ferramentas que viabilizam esta análise.

A coleta dos dados para o estudo de caso foi realizada durante a Copa do Mundo FIFA Brasil 2014², tendo sido iniciada no dia 09 de Junho de 2014 (três dias antes do jogo inaugural da competição) e finalizada no dia 14 de Julho de 2014 (um dia após a final do evento). Foram coletados dados durante os dias úteis no período da manhã e por um intervalo de, aproximadamente, 3 horas. Isto resultou em 21 dias de coleta de dados. Tal restrição no período de

\footnotetext{
1Para os autores, um "aspecto" correspondeu a um tema de governo, como saúde ou educação.

${ }^{2} \mathrm{http}: / /$ www.fifa.com/worldcup
} 
coleta foi estabelecida por questões operacionais; mas, não foi considerada um problema para o alcance dos resultados estabelecidos. Vale ressaltar que a restrição no período de coleta não prejudicou o alcance dos resultados traçados para o estudo de caso; apenas limitou a possibilidade de considerar as análises geradas definitivas para o assunto analisado.

Uma busca parametrizada, que correlacionou os temas 'Copa do Mundo' e 'Governo', foi utilizada. Os termos utilizados como critério de busca para cada tema são apresentados no Quadro 1. Foi estabelecido que seriam selecionadas para a coleta apenas as publicações no idioma português, que contivessem ao menos uma palavra-chave do tema "Copa do Mundo" e ao menos uma palavra-chave do tema 'Governo' (Quadro 1). Para a escolha das palavras chaves, foi feita uma consulta aleatória a publicações no Facebook e Twitter que mencionavam a palavra Copa (tema 'Copa do Mundo') e observadas hashtags e palavras mais citadas para o tema nestas publicações. Para o tema "Governo", foram selecionadas palavras relacionadas a grandes áreas de governo: educação, saúde, transporte e segurança, além da menção direta a governo e políticos.

Após a coleta, fez-se necessário tratar os dados obtidos para adequá-los para a etapa de análise, compatibilizando-os com os formatos utilizados pelas ferramentas de análise e visualização. Por este motivo, foi necessário desenvolver scripts customizados para converter os dados, originalmente em estrutura hierárquica $\mathrm{JSON}^{3}$, para um formato plano em $\mathrm{CSV}^{4}$, resultando em três arquivos.

Quadro 1: Termos utilizados na coleta de dados do Twitter e Facebook por tema.

\begin{tabular}{|l|l|}
\hline \multicolumn{1}{|c|}{ Tema } & \multicolumn{1}{c|}{ Palavras-chaves } \\
\hline Copa do Mundo & $\begin{array}{l}\text { '\#BoraBrasil', '\#VaiTerHexa', '\#NaoVaiTerCopa', '\#FanFest', '\#FanFestFIFA', 'copa do mundo', 'copa } \\
\text { da fifa', '\#PadraoFIFA', '\#PadrãoFifa', 'FIFA', '\#NãoVaiTerCopa', '\#VaiTerCopa', 'selecao brasileira', } \\
\text { 'seleção brasileira', 'brasil 2014', 'copa 2014' e 'copa' }\end{array}$ \\
\hline $\begin{array}{l}\text { Governo } \\
\text { 'metrô', 'ônibus', 'onibus', 'transporte', 'hospital', 'governo', 'governo federal', 'governo brasileiro', } \\
\text { 'politico', 'político', 'polícia', 'policia', 'seguranca', 'segurança', 'hospitais', 'saúde', 'saude', } \\
\text { 'educacao', 'educação' e 'governo' }\end{array}$ \\
\hline
\end{tabular}

O primeiro arquivo CSV continha informações referentes ao conteúdo publicado por um usuário, como por exemplo: data da publicação, texto publicado, login do usuário, quantidade de seguidores/amigos, localização e nível de influência do usuário nas redes sociais. O segundo arquivo CSV armazenava a relação entre os usuários e as hashtags utilizadas. O terceiro arquivo CSV correlacionava usuário e os usuários mencionados por ele nas publicações.

Outros quatro arquivos foram gerados para a visualização no formato de grafo da rede social. Dois arquivos representaram os vértices da rede: um com todos os usuários da rede; e o outro com as hashtags. Outros dois arquivos representaram as arestas dos grafos: o primeiro com a relação entre os usuários (pares com usuários e mencionados), e o segundo com a ligação entre hashtags utilizadas na mesma publicação.

\footnotetext{
${ }^{3}$ JSON é um acrônimo para JavaScript Object Notation, um formato para intercâmbio de dados computacionais. ${ }^{4} \mathrm{CSV}$ é um acrônimo para Comma-Separated Values, que é um arquivo tabular os dados (números e texto) no formato de texto.
} 
A partir destes arquivos, com os dados tratados, partiu-se para as fases de análise e visualização das informações, em um ciclo de refinamento das descobertas, que incluiu a análise visual dos dados. Os resultados mais relevantes são apresentados na seção "Resultados do Estudo de Caso".

\section{RESULTADOS E DISCUSSÃO}

Várias descobertas foram realizadas a partir da análise e visualização dos dados tratados. No geral, o estudo de caso, demonstrou que é possível conhecer o que está sendo discutido em redes sociais on-line, descobrir o sentimento de um usuário a respeito um tema, apontar evoluções ao longo do tempo e identificar influenciadores para um tema. Algumas destas descobertas são exemplificadas neste artigo.

\section{Evolução das Publicações ao Longo do Tempo}

Analisar a quantidade de publicações ao longo do tempo permite conhecer o quanto determinado tema está sendo comentado nas redes e é relevante para os usuários; ou quando um tema deixa de ser comentado. Um exemplo de como é possível observar a evolução de publicações ao longo do tempo é apresentado na Figura 1. A visualização da figura indica que as publicações que associavam os temas "Governo" e "Copa" diminuíram com o decorrer da Copa do Mundo; possivelmente graças ao envolvimento das pessoas com o evento e ao clima de euforia que se instalou pelo país. A maior concentração de publicações, relacionando os temas, ocorreu na semana inaugural do evento (marcações B, C, D, E, F). Após este período, dois outros dias se destacam em quantidade de publicações, 09 e 14 de Julho. Uma possível explicação para esta inversão é a reação dos usuários quanto ao desempenho da seleção brasileira ao final do mundial. Em $Q$, temse o dia posterior à derrota do Brasil para a Alemanha; e T representa o dia seguinte ao final do torneio.

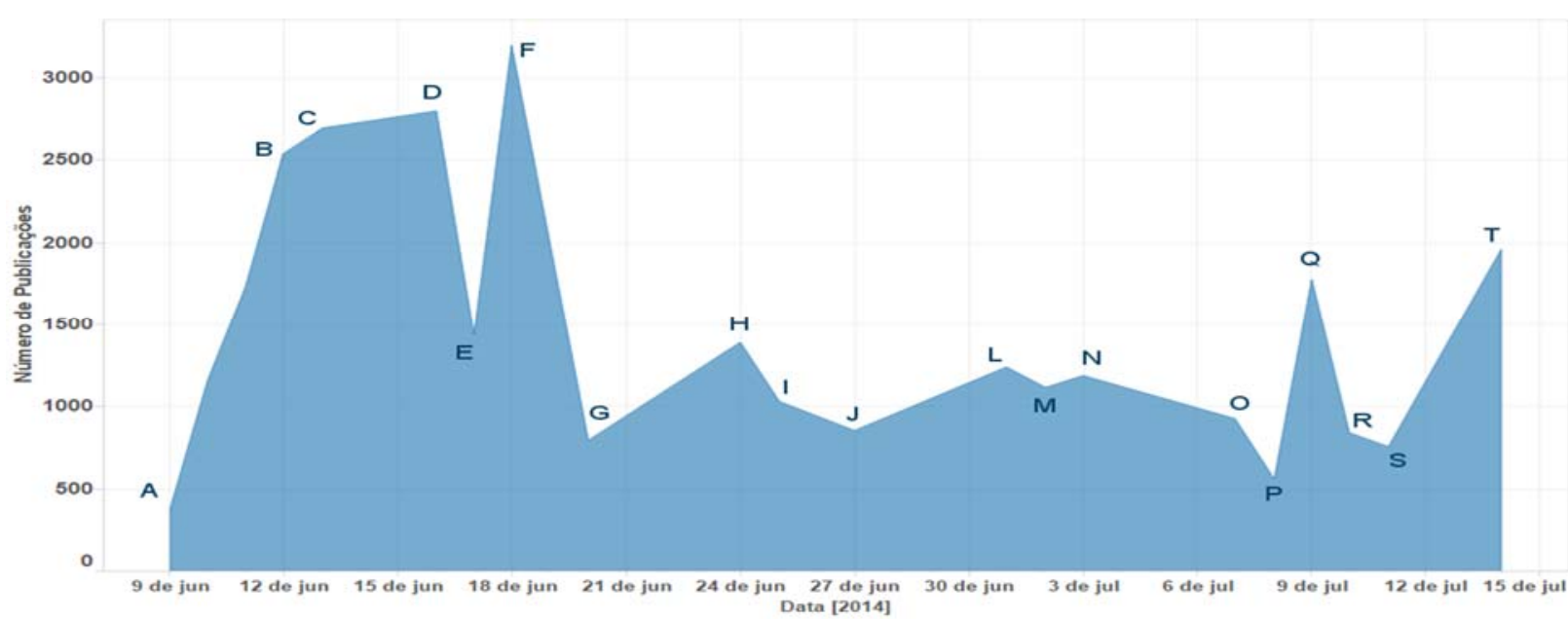

Figura 1: Número de publicações por dia. 


\section{Localização Geográfica das Publicações}

Além da visão cronológica, as localizações geográficas das publicações foram observadas; com uso de mapas. A maior parte das publicações coletadas - cerca de 97,95\% do universo - não forneceu a sua localização. Este é um comportamento conhecido em publicação em redes sociais, devido a preocupações com privacidade. Apesar do conjunto de dados ser menor, sua análise permite ter uma amostra de como foi a distribuição geográfica das publicações durante o evento.

No mapa à esquerda da Figura 2, observa-se que a maioria das publicações partiram dos Estados que foram sede de jogos da Copa do Mundo. Na figura, a cor representa o número de publicações em cada estado. Quanto mais escuro, mais publicações o estado possui. O mapa à direita da Figura 2 apresenta, por meio de círculos graduados, uma análise realizada da quantidade de publicações por cidade. Nota-se que a maior parte das publicações foram feitas nas grandes capitais do país, principalmente as que foram cidades sede da Copa do Mundo, com grande concentração no eixo Rio de Janeiro - São Paulo. O tamanho e a cor dos círculos representam o número de publicações.

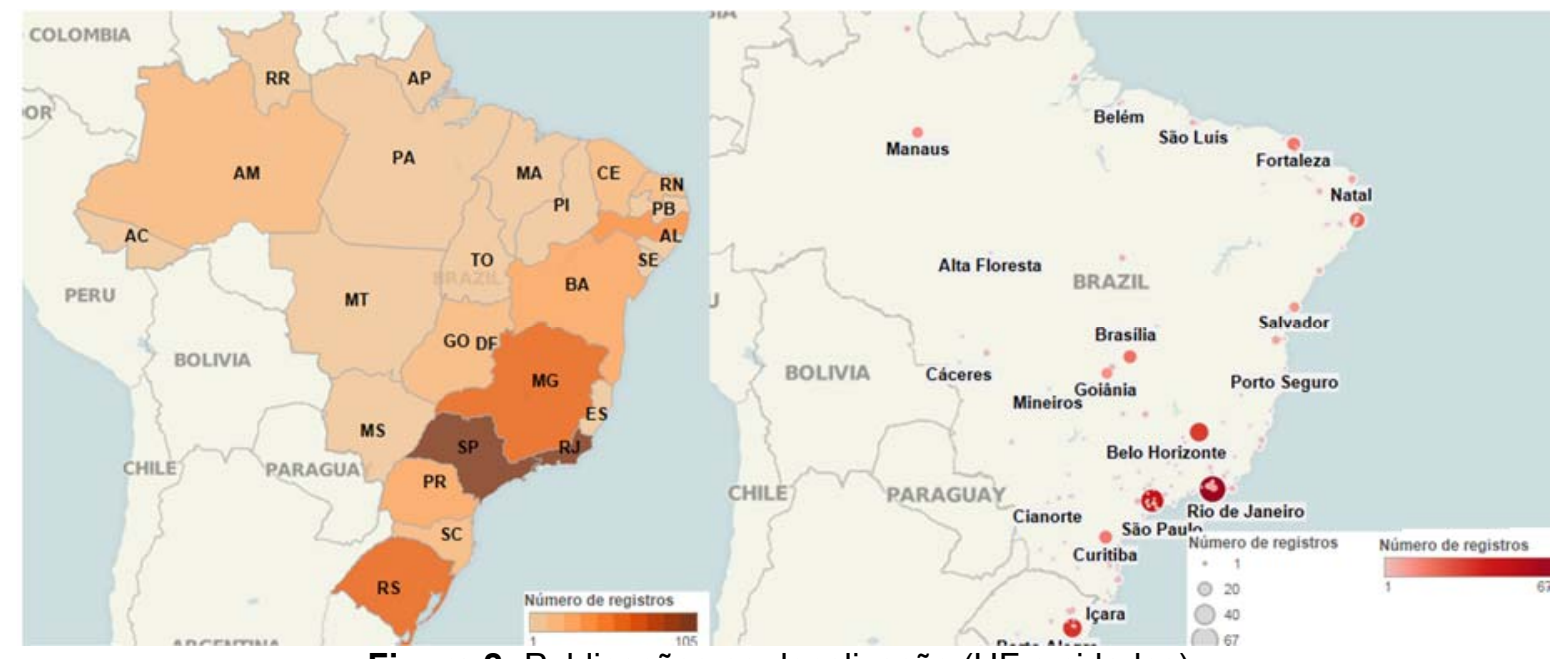

Figura 2: Publicações por localização (UF e cidades).

\section{Análise de conteúdo}

Em redes sociais, hashtags são utilizadas para contextualizar às publicações, segundo as boas práticas das mídias sociais (TWITTER, 2010). Entretanto, observa-se que são utilizadas pela imprensa também para indicar autoria da publicação. Ainda assim, a análise das hashtags permite ter uma noção aproximada do contexto das publicações e apoia a compreensão do que está sendo discutido e da evolução de um determinado tema.

Por exemplo, uma análise sobre a evolução do uso de hashtags durante o período é apresentada na visualização da Figura 3. Com o objetivo de melhorar a visualização para esta análise, apenas as seis hashtags mais citadas foram representadas no gráfico. $O$ eixo $Y$ da imagem exibe a quantidade de ocorrência de uma hashtag, e o eixo $\mathrm{X}$ os dias observados. A espessura de 
uma linha de hashtag apresenta o total acumulado ocorrências da hashtag durante todo o período. O gráfico evidencia que as hashtags 'copa' e 'copa2014' foram as mais usadas no período. É possível observar também que durante o início da copa, a hashtag 'naovaitercopa' foi mais utilizada, tendo seu pico de publicações na abertura da copa (12/06/2014). Logo após este período, o uso desta hashtag decaiu. Observa-se também um pico na hashtag 'copadascopas' no dia 24/06. Analisando os comentários relacionados à hashtag, é possível concluir um entusiasmo dos usuários em decorrência dos resultados negativos dos jogos das grandes seleções como por exemplo Espanha, Itália e Inglaterra.

Ainda em relação à análise de hashtags, um outro estudo foi realizado, desta vez, classificando-as manualmente em dois grupos: favorável e não favorável à copa. O grupo dos favoráveis incluiu as hashtags: 'copadascopas', 'vaitercopa', 'queromaiscopa', 'vivaobrasil', 'hexa', entre outras. O grupo contrário a copa reuniu as hashtags: 'copadavergonha', 'nãovaitercopa', 'vaitermanifestação', 'vergonhadasvergonhas', 'vazafifa', etc. Este exercício não pode ser tomado como uma análise de sentimento das publicações, já que o conteúdo completo não foi considerado. Ainda assim, aponta para uma inclinação em apoiar, ou não, a copa do mundo, já que cada hashtag indica o contexto da publicação. Neste cenário, e levando em consideração o período total da observação, 53,25\% das publicações foram favoráveis à copa.

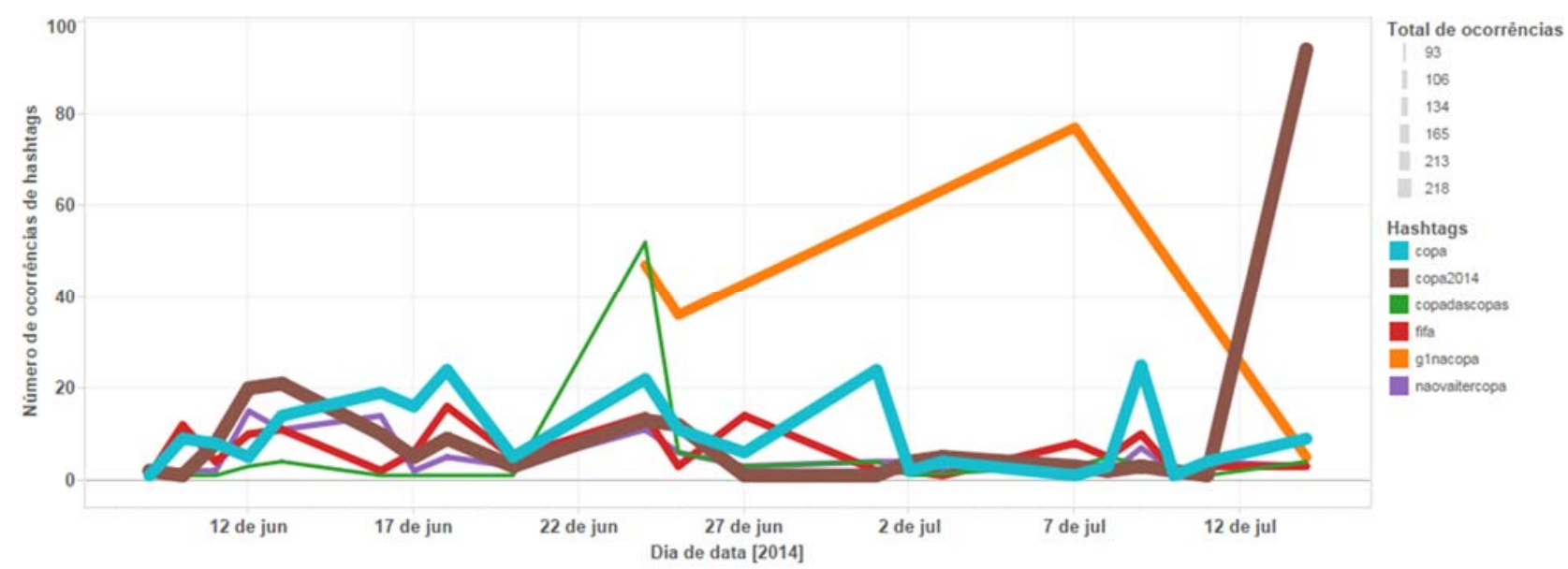

Figura 3: Hashtags mais utilizadas durante o período observado.

Considerando sua importância para contextualizar as publicações, as hashtags também foram agrupadas por temas (ou aspectos) relacionado ao Governo. Este agrupamento é apresentado no Quadro 2.

Quadro 2: Grupos temáticos de hashtags.

\begin{tabular}{|l|l|}
\hline Grupo & Exemplos de hashtags associadas aos grupos \\
\hline Política & $\begin{array}{l}<\text { Muitas hashtags relacionadas a política citam explicitamente nomes de partidos e candidatos. Como } \\
\text { o artigo foi submetido durante a campanha eleitoral, tais hashtags foram suprimidas.> }\end{array}$ \\
\hline Manifestações & $\begin{array}{l}\text { acordabrasil, copadasparalisações, mudabrasil, manifestações, ogiganteacordou, vemprarua, } \\
\text { lutarnãoécrime, acordameupovo, vaitermanifestacao, protestos, nacopatemluta }\end{array}$ \\
\hline Transporte & aeroporto, metrô, aquinaotemonibus, mobilidadeurbana, passelivre, transporte \\
\hline Educação & $\begin{array}{l}\text { copadascotas, educação, queromaisensinodequalidade, podetercopacomeduca, } \\
\text { queromaisuniversidadesfederais, escola, professor }\end{array}$ \\
\hline
\end{tabular}




\begin{tabular}{|l|l|} 
Segurança & copadocrime, crisenapf, euconfionapf, pfnacopa, segurança, violência \\
\hline Saúde & $\begin{array}{l}\text { crisenosus, maishospitais, maismedicos, maissaúde, queromaishospitais, } \\
\text { nãosefazcopacomhospitais, gesaoemsaude, hospitais, necessitadosdasaude }\end{array}$ \\
\hline
\end{tabular}

Com base neste agrupamento, foi possível categorizar as publicações coletadas, obtendo assim os temas mais mencionados por usuários das redes Twitter e Facebook - dentro do universo de publicações coletadas neste trabalho e que continham ao menos uma hashtag. Tal categorização é apresentada na Figura 4, por meio de uma visualização do tipo bolhas de ar. Cada bolha representa um grupo (categoria) e o seu tamanho indica o respectivo número de publicações associadas a este grupo. Observa-se que a maior quantidade de registros abordou o tema "política", seguido do tema "manifestações" e "segurança". Os demais temas foram comentados com menor frequência. Observa-se ainda que o tema saúde foi mais citado do que o tema educação, por exemplo.

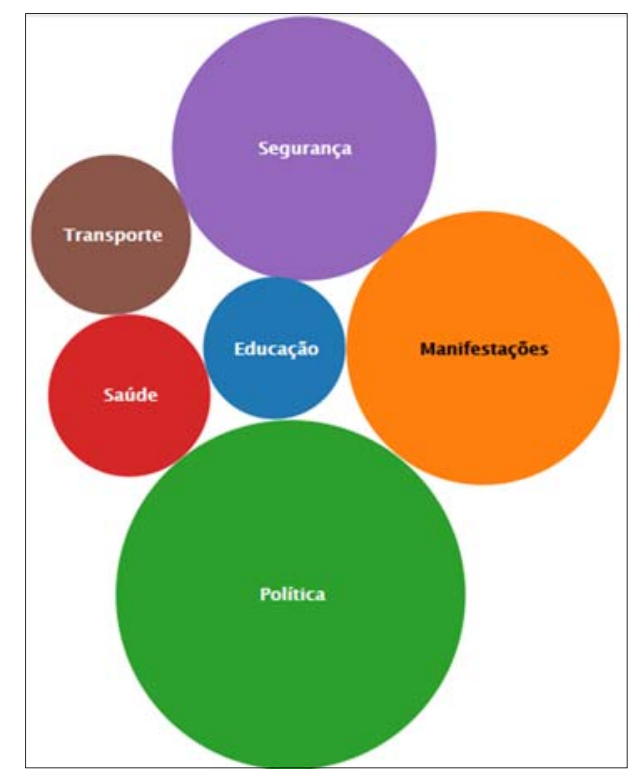

Figura 4: Publicações por grupo de assuntos de hashtags.

Ainda em relação aos temas mais discutidos durante o período de observação, buscou-se também identificar a evolução das menções aos temas ao longo da Copa. Esta análise pode ser visualizada na Figura 5 através de um gráfico de linhas - no qual o eixo $X$ representa o período de tempo; o eixo Y, a quantidade diária de publicações; e a espessura da linha representa a quantidade acumulada total de publicações durante o período de coleta. É interessante notar que as discussões a respeito dos temas, apresentado no gráfico, seguem um determinado padrão. Exceto pelos dias 17/06, 24/06 e 09/07, nos quais, respectivamente, os temas "manifestações", "segurança" e "saúde" se distanciaram do padrão apresentado no período completo. Essas distorções foram provocadas, possivelmente, por: (i) no dia 17/06 ocorreu um significativo protesto "anti-copa", horas antes do jogo Brasil e México, em Fortaleza, no qual houve confronto entre os policiais manifestantes 5 ; (ii) no dia 24/04 existiram diversos comentários com relação à ameaça de greve na polícia federal e suas ações durante a copa; e (iii) no dia 09/07 as redes sociais foram bastante utilizadas para criticar a 
seleção brasileira - após a derrota para a Alemanha - dentre essas críticas, as que tiveram maior número de compartilhamento, além das relacionadas com o grupo política, foram as relacionadas ao investimento do governo em saúde.

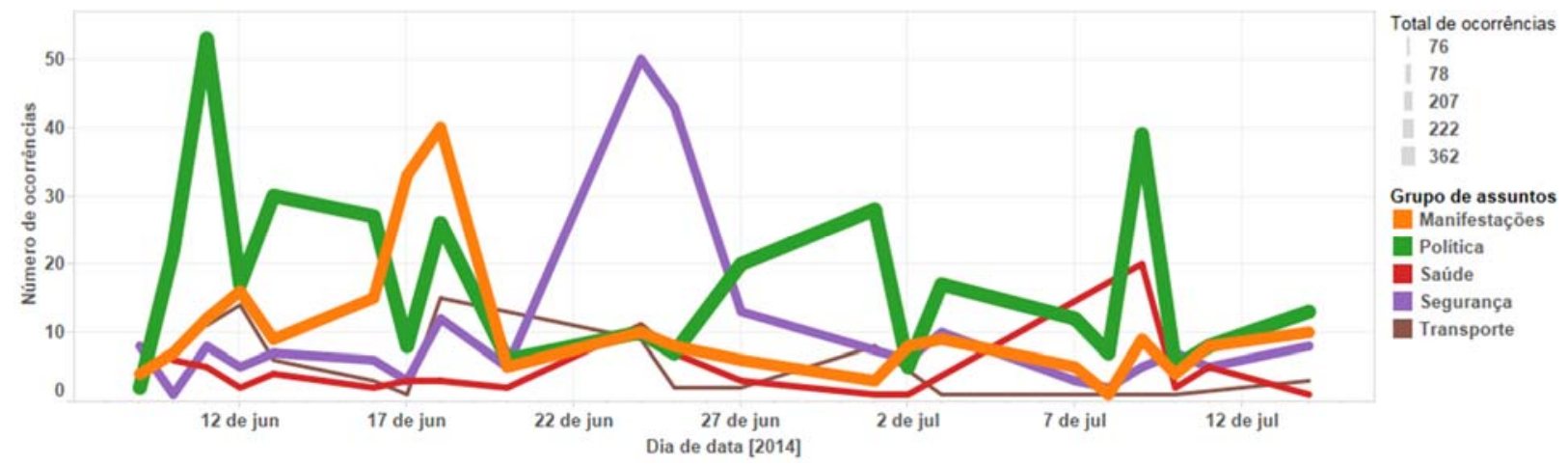

Figura 5: Grupo de assuntos por dia.

Outra análise realizada foi relacionar os temas das publicações com hashtags ao perfil dos usuários, com o objetivo de identificar, por exemplo, se a prioridade dos assuntos discutidos era a mesma para homens e mulheres (Figura 6).

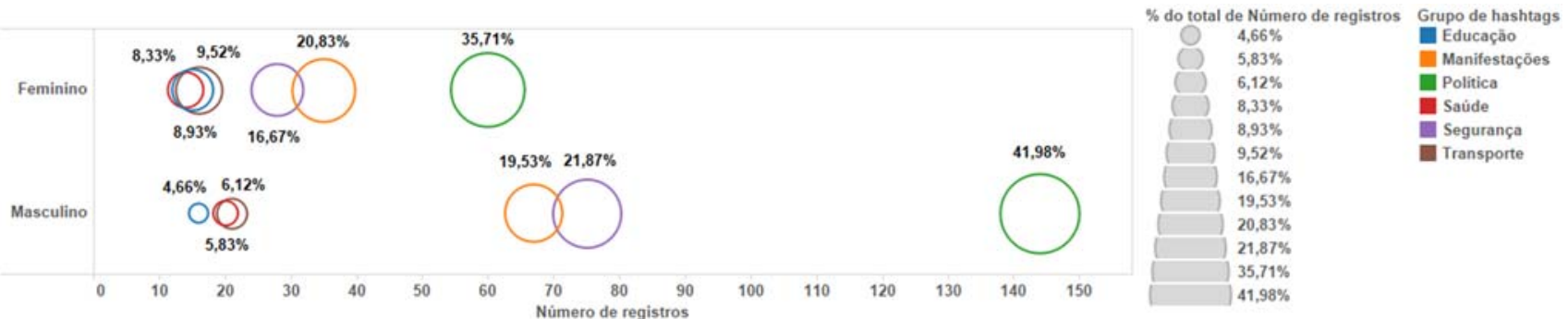

Figura 6: Grupo de assuntos por gênero.

A Figura 6 retrata os temas mais comentados por cada um dos gêneros. O eixo $Y$ representa o gênero; o eixo $\mathrm{X}$, o número de publicações; a cor do círculo representa a categoria; e o tamanho do círculo representa a porcentagem das publicações da categoria em relação a todas as publicações analisadas para o gênero. É possível notar que o número de publicações feitas por homens é maior que o de publicações feitas por mulheres para todas as categorias. Também é interessante observar que as temáticas de governo mais comentadas são muito parecidas por gênero; com exceção da temática "segurança" que, para o sexo masculino, teve um número de publicações maior que a temática "manifestações", por sua vez, mais comentada pelas mulheres.

Importante destacar que, assim como a localização, a informação sobre o gênero não é compartilhada por muitos dos usuários. No entanto, o universo de publicações com que continham o sexo foi bem maior: $49,5 \%$ dos perfis trazem esse dado. Apesar do número de publicações analisadas ser inferior ao total de publicações, a visualização demonstra que descobertas interessantes podem ser feitas ao analisar as publicações considerando características do perfil. Adicionalmente, técnicas de inferência de sexo (MIRANDA et al., 2014) podem ser utilizadas para aumentar a abrangência da análise. 


\section{Análise de Redes - Usuários, Hashtags e Influenciadores}

A rede de usuários e menções foi analisada a partir de um grafo dirigido, onde o grau de entrada representa as menções que o usuário recebeu e o grau de saída representa as menções feitas. A Figura 7 apresenta a rede dos usuários mais mencionados. As cores representam as comunidades (calculada através de uma funcionalidade da aplicação). O grafo exibe os principais mencionados e todos os usuários que os mencionaram. Isto significa que - durante o período de coleta - estes usuários foram mais mencionados ou tiveram suas publicações compartilhadas mais vezes. O tamanho do nó representa o grau de entrada do usuário (quantidade de menções que o usuário recebeu). É possível observar que a maioria dos grupos que existem são isolados, ou seja, existem poucas ligações entre os usuários mais mencionados. Interessante notar aqui que usuários comuns, como o "F" e o "B", foram mais mencionados do que grandes canais de comunicação (ex: nós "E" e "J").

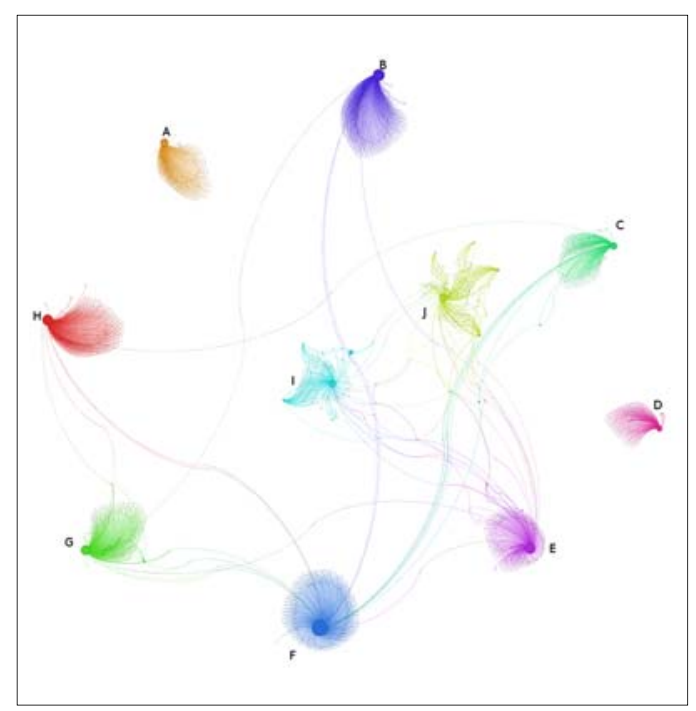

Figura 7: Usuários mais mencionados.

Especificamente para cinco usuários comuns mais mencionados, verificou-se que as publicações mais mencionadas usaram a ironia, relacionando os temas ' Copa do Mundo', 'Saúde' e 'Educação'. Como exemplo, a publicação do usuário "F" mais compartilhada (que aumentou consideravelmente seu "grau de entrada") tinha o seguinte conteúdo: "a copa é tão maravilhosa que fez o schumacher acordar pra ver o jogo da alemanha isso é a prova de q nao precisa de hospital qnd se tem copa". Esta publicação é de 16 de Junho de 2014 - dia em que a seleção da Alemanha estreou no mundial com vitória expressiva sobre Portugal. No mesmo dia, o ex-piloto de Fórmula 1 Michael Schumacher saiu do estado de coma, no qual se encontrava havia seis meses.

A Figura 8 apresenta um grafo no qual as hashtags são os nós e as arestas representam uma ligação entre hashtags utilizadas numa mesma publicação, ou seja, hashtags contidas em uma mesma publicação possuem uma aresta em comum. Foi utilizado um filtro para serem exibidas apenas as ligações entre hashtags mais frequentes. A partir dessa visualização é possível observar os agrupamentos de hashtags com relação a determinados assuntos. É interessante notar a grande 
quantidade de coocorrência entre as hashtags: 'fifa', 'naovaitercopa', 'brazil', 'não vai ter copa', 'anonymous' e 'não vai ter copa'. As hashtags que faziam referência a partidos políticos ou candidatos a eleição presidencial estão descaracterizadas no gráfico, em função do período eleitoral. Estas hashtags ocorreram muitas vezes em publicações que também continham a hashtag "g1naCopa". As hashtags "g1nacopa" e "acordabrasil" são as hashtags com os maiores números de conexões com outras. Para o universo de dados coletados, a hashtag "g1nacopa" foi bastante citada, o que aponta para a oportunidade de analisar mais detalhadamente a influência da grande imprensa nas discussões de redes sociais on-line.

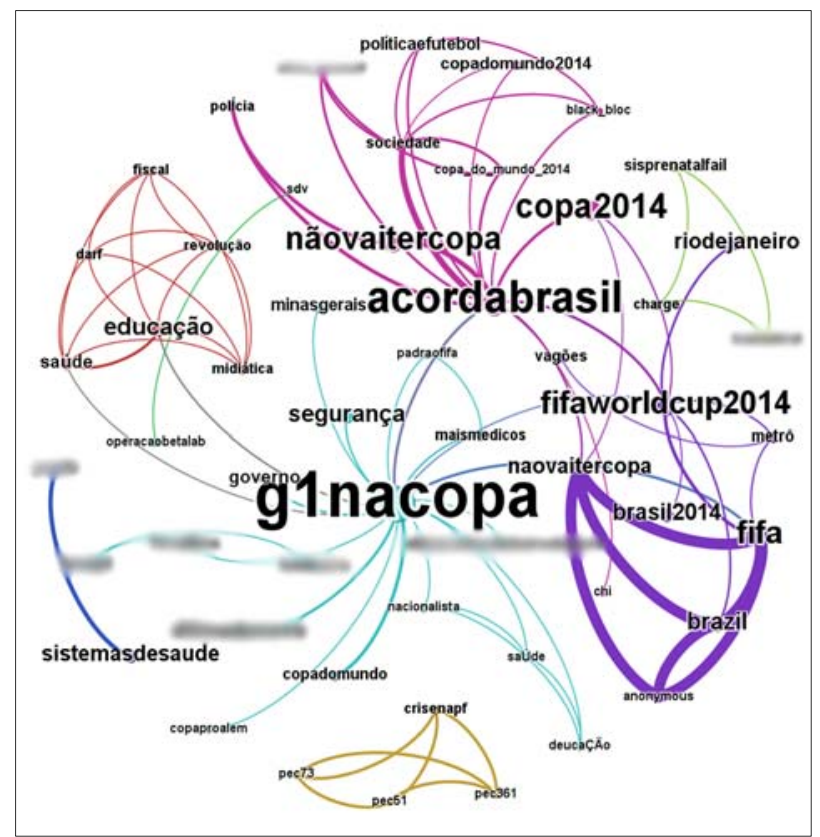

Figura 8: Grafo de hashtags.

\section{CONCLUSÕES}

O estudo de caso conduzido aponta para a viabilidade de utilizar análise de rede social (social analytics) para conhecer o que está sendo falado pelos cidadãos e aproximar o governo da sociedade. Dentre as dificuldades encontradas na condução do trabalho, pode-se citar: (1) calibrar as palavras-chave para maximizar a cobertura dos comentários relevantes e, ao mesmo tempo, prefiltrar publicações não relacionadas, controlando custo de acesso a informação e facilitando o trabalho de limpeza. Esta calibragem também é importante para aumentar a confiança de que a análise foi feita sobre dados que representam uma cobertura adequada das publicações; (2) identificar o quanto a análise dos dados de uma rede social é uma boa representação da realidade, por exemplo refletindo os diferentes grupos da sociedade. Isto é particularmente importante para as análises no âmbito governamental.

Todas as etapas de análise de redes sociais - coleta, tratamento, análise e apresentação foram exercitadas. O tratamento de dados mostrou que é importante organizar os dados em formatos específicos para as ferramentas de análise e normalizá-los. As análises sobre os dados coletados mostraram que é possível obter insights significativos sobre determinado tema. Mostrou 
também que o processo de análise exige desenvolver um conhecimento aprofundado sobre técnicas de visualização e adequação destas técnicas para o tipo de dado analisado.

Técnicas de análise foram exercitadas durante o estudo de caso, apontando para informações que não eram conhecidas a priori. Ressalta-se, porém, que como foram estabelecidas restrições para a coleta de dados, as análises apresentadas não podem ser consideradas conclusivas para o tema. Ainda assim, os objetivos do estudo de caso foram atingidos e ficou demonstrado que é viável utilizar publicações de redes sociais para descobrir o sentimento de um usuário sobre um tema, apontar tendências, identificar os temas mais comentados e os usuários influenciadores sobre um tema.

Como trabalho futuro, uma vez constatada a viabilidade de utilizar dados de redes sociais e identificadas as ferramentas e técnicas que podem ser utilizadas, será escolhido um programa de governo específico para fazer uma análise aprofundada sobre o sentimento dos cidadãos sobre este programa. Para este trabalho, serão exploradas também técnicas de análise de sentimentos e de mineração de texto. Com mineração de texto, e em particular mineração visual de texto (LOPES et al., 2007), será possível ampliar a análise de conteúdo para considerar todo o texto da publicação e não apenas as hashtag.

\section{REFERÊNCIAS}

FRANÇA, T.; OLIVEIRA, J.. Análise de sentimento de tweets relacionados aos protestos que ocorreram no brasil entre Junho e Agosto 2013. Brazilian workshop on social network analysis and mining, 2014.

INSELBERG, A.. Visual data mining with parallel coordinates. Computational Statistics, v.13, 1998.

LOPES, A.; PINHO, R.; PAULOVICH, F.; MINGHIM, R. Visual text mining using association rules. Comput. Graph.7), p.316-326. 2007.

MIRANDA, R.; CARVALHO, A.; PAPPA, G.. Influência de sexo e idade de usuários no Twitter. Brazilian workshop on social network analysis and mining, 2014.

SAPIRAS, L.; BECKER, K. Identificação de aspectos de candidatos eleitorais em comentários de notícias. Brazilian workshop on social network analysis and mining, 2014.

SLOCUM, T.; MCMASTER, R.; KESSLER, F.; HOWARD, H.. Thematic Cartography and Geovisualization. USA - Prentice Hall Series in Geographic Information Science, 3 ed., 2008.

THEODORO, I.; RANGEL, F.; FRANÇA, T.; OLIVEIRA, J.. Análise dos influenciadores dos protestos brasileiros de 2013 via Twitter. Brazilian workshop on social network analysis and mining, 2014.

VLIEGEN, R.; VAN WIJK, J.; VAN DER LINDEN, E.. Visualizing business data with generalized treemaps. Visualization and Computer Graphics, IEEE Transactions on, v.12, n.5, p.789-796, 2006.

WASSERMAN, S.; FAUST, K.. Social Network Analysis. Cambridge: Cambridge University Press, 1994. 